\title{
Annular Pancreas with a Duodenal Web: a Rare Presentation with Simultaneous Intrinsic and Extrinsic Duodenal Obstruction
}

\author{
Deepa Makhija, Hemanshi Shah, Vikrant Kumbhar, Kiran Khedkar \\ Department of Paediatric Surgery, TNMC \& BYL Nair Hospital, Mumbai, India
}

Congenital duodenal obstruction is a rare cause of neonatal intestinal obstruction caused by various intrinsic and extrinsic congenital lesions. Annular pancreas is one of the causes of extrinsic duodenal obstruction and a duodenal web is one of the causes of intrinsic duodenal obstruction. The simultaneous occurrence of an extrinsic and intrinsic pathology is rare. Only four such cases have been reported in literature. We present a similar case of male neonate with partial duodenal obstruction caused by annular pancreas and an intrinsic duodenal web.

Korean J Pancreas Biliary Tract 2016;21(4):199-201

\author{
Received May 6, 2016 \\ Revised Jun. 15, 2016 \\ Accepted Jul. 5, 2016
}

Corresponding author: Hemanshi Shah

Department of Paediatric Surgery, TNMC \& BYL Nair Hospital, Mumbai, Maharashtra 400008, India

Tel. +91-9930099029, +91-0223027324

Fax $+91-02223072663$

E-mail; hemanshisshah@gmail.com

Keywords: Congenital, Duodenal obstruction, Annular pancreas

This is an Open Access article distributed under the terms of the Creative Commons Attribution Non-Commercial License (http:/ creativecommons.org/licenses/by-nc/3.0/) which permits unrestricted non-commercial use, distribution, and reproduction in any medium, provided the original work is properly cited.

Copyright (C) 2016 by The Korean Journal of Pancreas and Biliary Tract

\section{INTRODUCTION}

Congenital duodenal obstruction is one of the causes of neonatal intestinal obstruction affecting approximately one in 2500 to 10,000 live births. ${ }^{1}$ Several intrinsic and extrinsic congenital lesions can lead to complete or partial obstruction of the duodenum. Annular pancreas is a developmental anomaly of the pancreas consisting of a ring of pancreatic tissue encircling the descending portion of the duodenum and forms an extrinsic cause of duodenal obstruction. ${ }^{2}$ About $4 \%$ cases can have a distal obstruction. ${ }^{3}$ Duodenal atresia is an intrinsic cause of duodenal obstruction. Type I duodenal atresia or membranous duodenal obstruction (mucosal diaphragm, or web): accounts for $92 \%$ of all cases and usually occurs in the second part of the duodenum. Presence of an intrinsic and extrinsic pathology simultaneously is rare. Only four such cases have been reported in literature. We present a case of male neonate with simultaneous extrinsic and intrinsic duodenal obstruction. ${ }^{4}$

\section{CASE}

A full term male neonate, delivered at an outer hospital, had history of bilious vomiting on day 3 and day 5 of life for which an erect abdominal $\mathrm{x}$ ray and ultrasound were done and found to be normal. Patient was accepting feeds and passing milk (yellow) stools. At the age of 12 days, patient was referred for multiple episodes of bilious vomiting.

On examination, there was minimal upper abdominal distention. Nasogastric tube had bilious aspirates. Erect radiography after instilling air through the nasogastric tube was suggestive of distended stomach with few specks of air in the distal bowel. An upper gastrointestinal tract contrast study showed grossly dilated stomach and upper part of duodenum with free passage of contrast in the distal bowel (Fig. 1). Ultrasound of the abdomen suggested dilated stomach and upper part of duodenum with collapsed bowel loops distally. A presumptive diagnosis of partial duodenal obstruction was made.

Intraoperatively, annular pancreas was seen (Fig. 2). Kimu- 


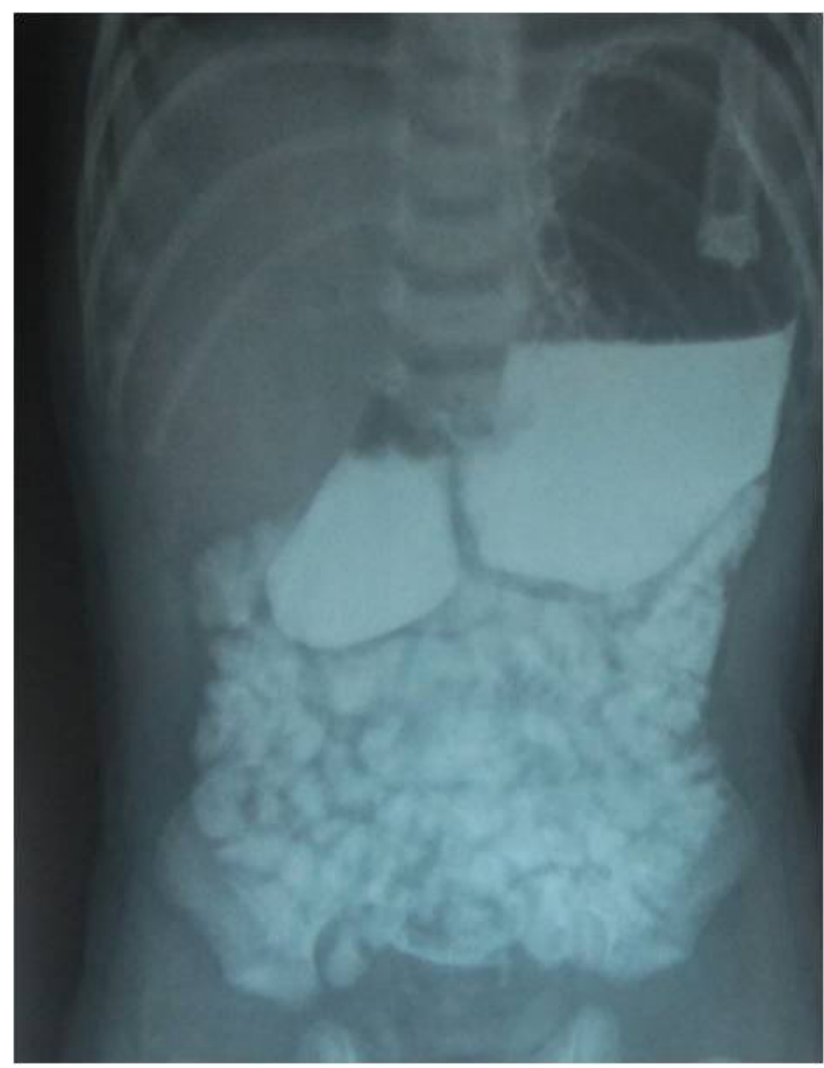

Fig. 1. Upper gastrointestinal tract contrast study showing dilated stomach and upper part of duodenum with free passage of contrast in the distal bowel.

ra's duodenoduodenostomy was planned. There was diffulty in placement of the transanastamotic feeding tube. The tube could not be negotiated into the third part of duodenum raising the suspicion of a distal obstruction. On extending the longitudinal incision over the distal limb, a duodenal web was seen (Fig. 3). The web was excised and duodeno-duodenostomy was completed over a transanastamotic tube. A large bore nasogastric tube was kept for gastric decompression.

Postoperatively, patient was started on enteral feeds through the transanastamotic tube. Oral feeds were started after a week. Histopathology report of the specimen was consistent with a duodenal web. Patient is doing well on follow up and has adequate weight gain.

\section{DISCUSSION}

Duodenal web and annular pancreas are both considered in the differential diagnosis of neonatal duodenal obstruction.

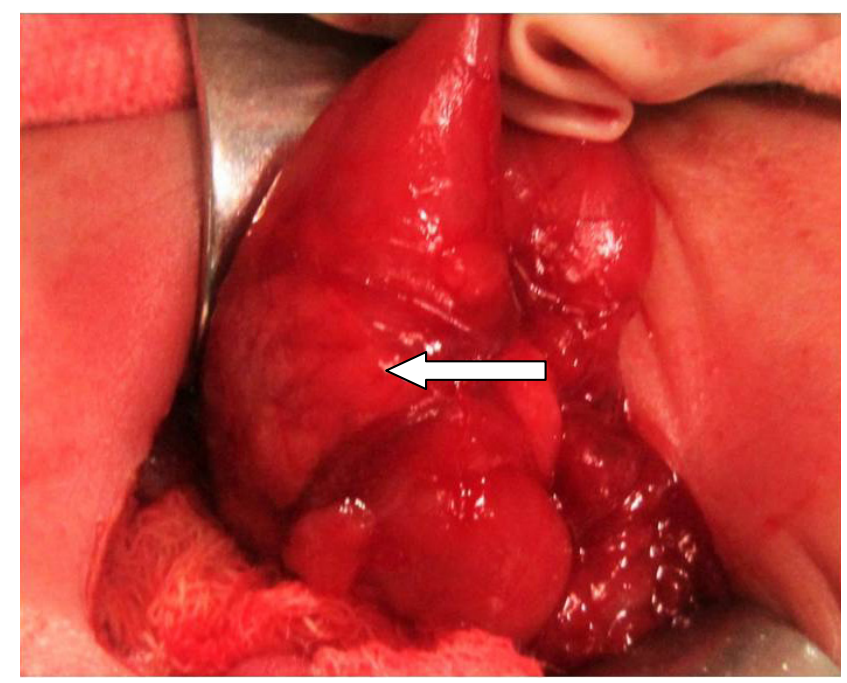

Fig. 2. Intra-operative image showing annular pancreas (arrow).

The simultaneous occurrence of a duodenal web and an annular pancreas is exceedingly rare. ${ }^{5}$

Annular pancreas is an uncommon congenital condition. It is estimated that it occurs in one of every 12,000-15,000 live births. ${ }^{6}$ The pancreas is normally formed from the fusion of the dorsal and ventral pancreatic buds between the first 4-8 weeks of embryonic life. Annular pancreas results due to failure of the ventral bud to rotate. It then elongates to encircle the upper part of the duodenum. Five theories have been suggested to explain the pathogenesis of annular pancreas, although various abnormalities appear to be involved in the developmental process ${ }^{2}$ - Hypertrophy of both dorsal and ventral primordium; Persistence and enlargement of the left bud of the paired ventral primordium; Fixation of the right bud of the ventral primordium prior to rotation; Adherence of the right ventral pancreatic bud to the duodenum; and Adherence of the tip of the left ventral angle to the duodenum.

Attachment of the ventral pancreas to the duodenal wall leaves a band of pancreatic tissue encircling the second part of the duodenum with varying degrees of underlying obstruction. There is controversy as to whether the annular pancreas plays a role in obstruction. It is generally accepted that the abnormally located pancreatic tissue is a visible indicator of an underlying duodenal abnormality that can range from minimal duodenal stenosis to atresia. ${ }^{7}$

Only anecdotal cases of duodenal obstruction having an- 


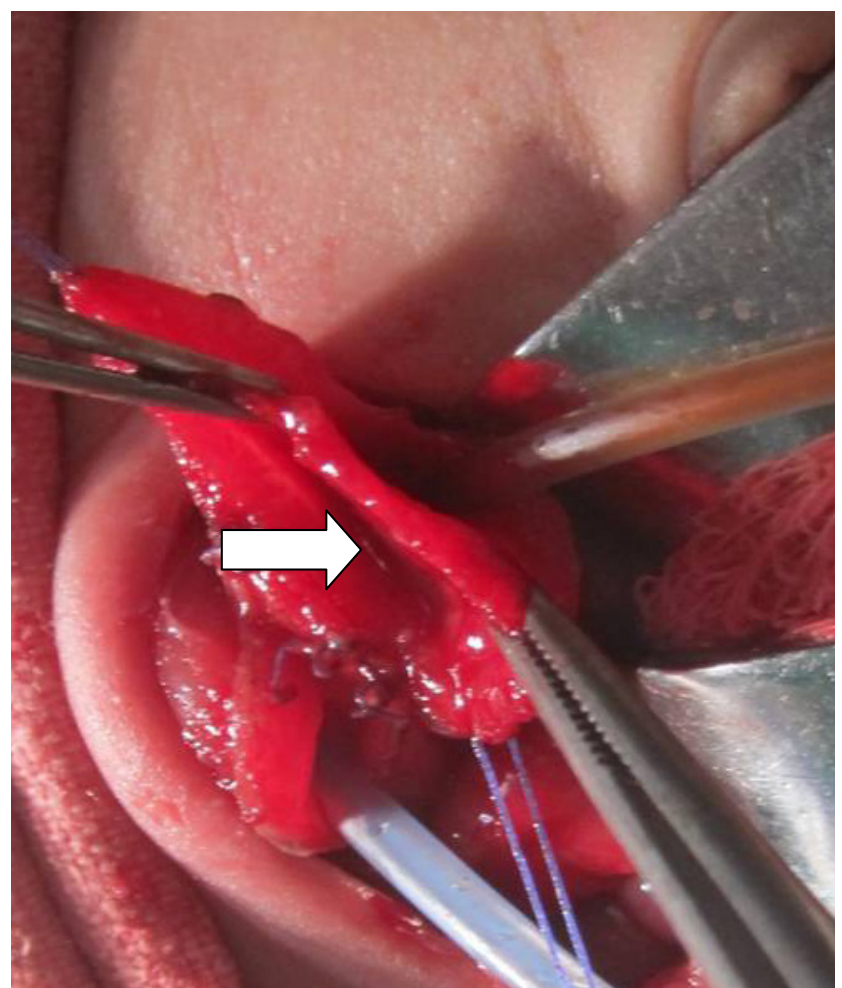

Fig. 3. Intra-operative image showing duodenal web causing intrinsic obstruction (arrow).

nular pancreas with distal web have been reported. Norton et al in 1991 reported sonographic demonstration of annular pancreas with web distally. ${ }^{5}$ Papandreau et al. in 2004 reported two cases of annular pancreas with membranous web distally. ${ }^{3}$ Arena et al. in 2008 reported a case of annular pancreas with wind sock web.

Preoperative imaging generally fails to diagnose an exact cause of the duodenal obstruction. The diagnosis is invariably confirmed at laparotomy when an annular pancreas and a distal obstruction is present. The management involves a by- pass procedure preferably done by a Kimura's duodenoduodenostomy.

The combination of annular pancreas and distal duodenal web is extremely rare. Patency of the duodenum distal to the apparent obstruction should always be checked in order to avoid misdiagnosis or delayed diagnosis of this combination. This case highlights the same.

\section{Conflicts of Interest}

The author has no conflicts to disclose.

\section{REFERENCES}

1. Chen QJ, Gao ZG, Tou JF, et al. Congenital duodenal obstruction in neonates: a decade's experience from one center. World J Pediatrics 2014;10:238-244.

2. Alahmadi R, Almuhammadi S. Annular pancreas: a cause of gastric outlet obstruction in a 20-year-old patient. Am J Case Rep 2014;15:437-440.

3. Papandreou E, Baltogiannis N, Cigliano B, Savanelli A, Settimi A, Keramidas $D$. Annular pancreas combined with distal stenosis. A report of four cases and review of the literature. Pediatr Med Chir 2004;26:256-259.

4. Applebaum H, Sydorak R. Duodenal Atresia and Stenosis-Annular Pancreas. In: O'Neil JA Jr, Rowe MI, Grosfeld JL, Fonkalsrud EW, Coran AG, editors. Pediatric Surgery. 7th ed. p1051-1057, New York, Mosby, 2007.

5. Norton KI, Tenreiro R, Rabinowitz JG. Sonographic demonstration of annular pancreas and a distal duodenal diaphragm in a newborn. Pediatr Radiol 1992;22:66-67.

6. Husarić E, Hotić N, Halilbasić A, Rahmanović E, Suljendić S. Annular pancreas with duodenal stenosis and intestinal malrotation in two years girl - case report. Paediatrics Today 2014;10:47-50.

7. McCollum MO, Jamieson DH, Webber EM. Annular pancreas and duodenal stenosis. J Pediatr Surg 2002;37:1776-1777.

8. Arena F, Impellizzeri P, Scalfari G, et al. An uncommon case of associate intrinsic and extrinsic stenosis of the duodenum in newborn. Pediatr Med Chir 2008;30:212-214. 